(C) The Authors 2014. This is an Open Access article, distributed under the terms of the Creative Commons Attribution licence (http:// creativecommons.org/licenses/by/3.0/), which permits unrestricted re-use, distribution, and reproduction in any medium, provided the original work is properly cited.

\title{
Breakfast consumption is positively associated with nutrient adequacy in Canadian children and adolescents
}

\author{
Susan I. Barr ${ }^{1 *}$, Loretta DiFrancesco ${ }^{2}$ and Victor L. Fulgoni $\mathrm{III}^{3}$ \\ ${ }^{1}$ Department of Food, Nutrition and Health, University of British Columbia, 2205 East Mall, Vancouver, BC, \\ Canada V6T $1 Z 4$ \\ ${ }^{2}$ Source! Nutrition, Toronto, ON, Canada \\ ${ }^{3}$ Nutrition Impact LLC, Battle Creek, MI, USA \\ (Submitted 11 October 2013 - Final revision received 14 May 2014 - Accepted 6 July 2014 - First published online 8 September 2014)
}

\section{Abstract}

Although breakfast is associated with more favourable nutrient intake profiles in children, limited data exist on the impact of breakfast on nutrient adequacy and the potential risk of excessive intakes. Accordingly, we assessed differences in nutrient intake and adequacy among breakfast non-consumers, consumers of breakfasts with ready-to-eat cereal (RTEC) and consumers of other types of breakfasts. We used cross-sectional data from 12281 children and adolescents aged 4-18 years who took part in the nationally representative Canadian Community Health Survey, 2004. Mean nutrient intakes (obtained using a multiple-pass $24 \mathrm{~h}$ recall method) were compared among the breakfast groups using covariate-adjusted regression analysis. Usual nutrient intake distributions, generated using the National Cancer Institute method, were used to determine the prevalence of nutrient inadequacy or the potential risk of excessive intakes from food sources alone and from the combination of food plus supplements. Of these Canadian children, $10 \%$ were breakfast non-consumers, $33 \%$ were consumers of RTEC breakfasts and $57 \%$ were consumers of other types of breakfasts. Non-consumption of breakfast increased with age ( $4-8$ years: $2 \%$; 9-13 years: 9\%; 14-18 years: 18\%). Breakfast consumers had higher covariate-adjusted intakes of energy, many nutrients and fibre, and lower fat intakes. The prevalence of nutrient inadequacy for vitamin $\mathrm{D}, \mathrm{Ca}, \mathrm{Fe}$ and $\mathrm{Mg}$ (from food alone or from the combination of food plus supplements) was highest in breakfast non-consumers, intermediate in consumers of other types of breakfasts and lowest in consumers of RTEC breakfast. For vitamin A, P and Zn, breakfast non-consumers had a higher prevalence of nutrient inadequacy than both breakfast groups. The potential risk of excessive nutrient intakes was low in all groups. Efforts to encourage and maintain breakfast consumption in children and adolescents are warranted.

\section{Key words: Dietary surveys: Nutrition assessment: Breakfast: Population studies}

The importance of breakfast in contributing to the nutrient intakes of children and adolescents has been recognised for decades, and has been the topic of numerous reviews ${ }^{(1-4)}$. These reviews and studies published more recently ${ }^{(5-7)}$ have indicated that breakfast consumption is frequently associated with higher energy and nutrient intakes, and that the overall nutrient profile appears most favourable among those who consume breakfasts that include ready-to-eat cereal (RTEC). Although most studies have been conducted in the USA, research conducted with children from other countries has yielded similar findings ${ }^{(6,8-11)}$. Despite these positive associations of breakfast consumption with nutrient intake, $20 \%$ of 9- to 13-year-old children and $32 \%$ of 14- to 18-year-old adolescents studied in the 1999-2006 National Health and Nutrition Examination Survey did not eat breakfast ${ }^{(5)}$, and the frequency of breakfast skipping has increased between the 1960s and the 1990s ${ }^{(12)}$.

It is probable that in many cases, higher nutrient intakes associated with breakfast and RTEC consumption translate to improved nutrient adequacy (where adequacy is defined as meeting nutrient requirements). Nevertheless, it is conceivable that higher nutrient intakes might have no impact on adequacy (if intakes of almost everyone met or exceeded the requirements) and could even increase the potential risk of adverse effects from excessive intakes. However, to date, most studies have not reported the association between breakfast consumption, or the type of breakfast consumed, and dietary nutrient adequacy as measured using the dietary reference intake (DRI) framework for dietary assessment ${ }^{(13)}$. Using this framework, the prevalence of dietary inadequacy can be

Abbreviations: AI, adequate intake; CCHS 2.2, Canadian Community Health Survey 2.2; DRI, dietary reference intake; EAR, estimated average requirement; RTEC, ready-to-eat cereal; UL, tolerable upper intake level.

*Corresponding author: S. I. Barr, fax +1 604822 5143, email susan.barr@ubc.ca 
estimated for most nutrients as the proportion of a population's usual nutrient intake distribution that falls below the estimated average requirement (EAR). If intakes are assessed accurately, one would expect that proportion of the population below the EAR to approximate the proportion of the population who do not meet their requirement for the physiological criterion of adequacy used to set the $\mathrm{EAR}^{(13)}$. Similarly, the prevalence of the potential risk of adverse effects from excessive nutrient intakes can be estimated as the proportion of the usual nutrient intake distribution that exceeds the tolerable upper intake level (UL). Because vitamin and mineral supplements may contribute to dietary adequacy as well as the potential risk of dietary excess ${ }^{(14-16)}$, it is important to account for supplement intake when assessing these parameters. Yet almost all studies examining the contribution of breakfast consumption have reported intakes from food sources alone. Accordingly, the purpose of the present study was to assess the differences in nutrient intake and adequacy among Canadian children and adolescents classified as breakfast non-consumers, consumers of breakfasts with RTEC and consumers of other types of breakfasts, considering intakes from food sources alone and from the combination of food sources and supplements. We hypothesised that breakfast consumption would be associated with a lower prevalence of nutrient inadequacy as assessed using the DRI framework, and would have little or no impact on the prevalence of the potential risk of excessive nutrient intakes.

\section{Methods \\ Data source and participants}

The present study used data from the Canadian Community Health Survey 2.2 (CCHS 2.2), a cross-sectional, nationally representative survey conducted by Statistics Canada in $2004^{(17,18)}$. The survey included a $24 \mathrm{~h}$ dietary recall, which was followed by a general health questionnaire assessing sociodemographic characteristics, physical activity, smoking, supplement use and food security, among other variables. Interviews were done by a proxy (parent or legal guardian) for children under the age of 6 years, with a parent or guardian (joint responses) for children aged 6 to 11 years, and by the respondent alone for individuals aged 12 years and above. In all cases, a parent or legal guardian consented to the participation of the child. Henceforth, 'respondent' or 'participant' is taken to include 'proxy' where appropriate. The target population for the survey was all individuals living in private dwellings in the ten Canadian provinces, which represented about $98 \%$ of the Canadian population. The sampling strategy was designed to be representative in terms of age, sex, geography and socio-economic status. The response rate for the survey was $76 \cdot 5 \%$, and a nonresponse adjustment was applied to the survey weights. Ethical approval for population health surveys conducted by Statistics Canada is based on the authority of the Statistics Act of Canada ${ }^{(17,19)}$. Approval to conduct the analyses reported herein was received from the Statistics Canada Research Data Centre Program ${ }^{(20)}$.
The present study included data from respondents aged 4 to 18 years who were not pregnant or lactating ( $n$ 12281). Breakfast consumption was self-reported and included any foods or beverages consumed at an eating occasion that the participant defined as breakfast during the $24 \mathrm{~h}$ dietary recall ${ }^{(18)}$. Those who did not report any items as breakfast were classified as breakfast non-consumers. Those who consumed RTEC as a component of breakfast were classified as RTEC-breakfast consumers, and those whose breakfasts did not include RTEC were classified as other-breakfast consumers.

\section{Nutrient intakes}

Dietary intake data were obtained using a slightly modified version of the US Department of Agriculture Automated Multiple-Pass Method for $24 \mathrm{~h}$ dietary recalls ${ }^{(18,21)}$. All respondents completed a $24 \mathrm{~h}$ dietary recall in person with a trained interviewer, and a subset of approximately $30 \%$ completed a second $24 \mathrm{~h}$ dietary recall by telephone 3 to $10 \mathrm{~d}$ later. Nutrient contributions from foods were based on data from the Canadian Nutrient File, version $2001 b^{(22)}$, which were primarily derived from the US Department of Agriculture Nutrient Database for Standard Reference $13^{(23)}$. The Canadian Nutrient File also includes Canadian-specific values for foods, including RTEC, which have differing composition in Canada than in the USA. In Canada, the nutrients that may be added to breakfast cereals (e.g. thiamin, niacin, vitamin $\mathrm{B}_{6}$, pantothenic acid, folate, $\mathrm{Mg}, \mathrm{Fe}$ and $\mathrm{Zn}$ ) and the amounts to be added are specified by the Food and Drugs Regulations ${ }^{(24)}$. For most nutrients, the amount that may be added is modest $(<10 \%$ of the daily value per serving), although it is somewhat higher for some nutrients (e.g. thiamin and Fe). In contrast, in the USA, voluntary fortification of breakfast cereals falls under food safety regulations and a fortification policy statement ${ }^{(25)}$. Generally, a wider array of nutrients and higher amounts (up to $100 \%$ of the daily value per serving) can be found in US breakfast cereal products.

The general health component of the CCHS 2.2 included questions on supplement use ${ }^{(18)}$. Respondents who had taken a vitamin or mineral supplement within the past month were classified as supplement users. These individuals were asked to locate their supplement containers (from which the interviewer recorded complete product information) and provided details on the frequency of supplement use during the past month. For each nutrient, the average daily amount consumed as a supplement was included as a variable in the CCHS 2.2 data file.

\section{Estimation of usual nutrient intake distributions}

Estimates of usual intake for each nutrient were determined using the National Cancer Institute method for a single dietary component $^{(26)}$, using data from the second $24 \mathrm{~h}$ dietary recall to adjust for within-individual variation. Based on intake on the day of the first $24 \mathrm{~h}$ dietary recall, separate usual intake distributions were generated for each nutrient by DRI age/sex group for breakfast non-consumers, RTEC-breakfast consumers and other-breakfast consumers. As has been described 
elsewhere $^{(27)}$, balanced repeated replication was used to create variance estimates, standard errors of percentiles and probabilities of meeting a DRI.

The prevalence of nutrient inadequacy was estimated as the proportion of respondents with usual intakes below the age/ sex-specific EAR for thiamin, riboflavin, niacin (as niacin equivalents), folate (as dietary folate equivalents (DFE, where $1 \mathrm{DFE}=1 \mu \mathrm{g}$ food folate or $0.6 \mu \mathrm{g}$ folic acid from fortified foods) $)^{(28)}$, Ca, P, Mg, $\mathrm{Zn}$, vitamin A (as retinol activity equivalents) and vitamins $\mathrm{B}_{6}, \mathrm{~B}_{12}, \mathrm{C}$ and $\mathrm{D}^{(13)}$. Vitamin $\mathrm{E}$ was not included in the CCHS 2.2 data file, as the 2001b version of the Canadian Nutrient File was substantially incomplete for this nutrient. The probability method was used for Fe, which does not have a symmetrical requirement distribution ${ }^{(29)}$; accordingly, we used the requirement distributions published by the Institute of Medicine ${ }^{(29)}$. The prevalence of the potential risk of excessive nutrient intakes was estimated as the proportion of respondents with usual intakes above the age/sex-specific UL for folic acid, Ca, P, Fe, Zn, and vitamins $\mathrm{B}_{6}$, $\mathrm{C}$ and $\mathrm{D}^{(13)}$. Vitamin $\mathrm{A}$, niacin and $\mathrm{Mg}$ were not examined for this analysis. The UL for vitamin A and niacin are based on preformed retinol and nicotinic acid or nicotinamide, respectively, and these nutrient forms are not available in the CCHS 2.2 data file. The UL for Mg is based on Mg added as a fortificant to foods and used as a supplement, but since $\mathrm{Mg}$ added to foods is also not available in the CCHS 2.2 data file, we could not assess intakes above the UL. Both nutrient inadequacy and the potential risk of excessive nutrient intakes were assessed for intakes from food sources alone and for total intakes from food sources plus supplements. The total intake distributions were generated for each breakfast group and age/sex group by adding each individual's daily supplemental intake of the selected nutrient (averaged over the past month) to their usual nutrient intake based on food sources alone ${ }^{(30)}$.

\section{Statistical analyses}

Statistical Analysis Software (version 9.3; SAS Institute, Inc.) and SUDAAN (version 10.0; RTI International) were used to analyse the data. All analyses were adjusted for the complex CCHS 2.2 sampling design using appropriate sample weights and the MISSUNIT option in SUDAAN due to a large number of cases where only one stratum within a primary sampling unit was encountered during balanced repeated replication. This option then calculates variance contribution using the difference from the overall mean of the population. Means, percentages and standard errors were obtained using the PROC DESCRIPT procedure. Covariate-adjusted mean nutrient intakes among the three breakfast groups were compared using regression analysis (i.e. using the PROC REGRESS procedure), with $P<0.05$ (Bonferroni-adjusted $P<0.0167$ ) to assess the significance of multiple comparisons. Covariates included in the analysis were energy intake, age, sex, race, food security, language spoken at home and supplement use. Physical activity and sedentary activity ('screen time') could not be included as covariates because they were not assessed in children under 6 years of age, and different assessment questions were used in those aged 6 to 11 years $v$. those 12 years and above.
Moreover, the age groups for activity assessment did not correspond to the DRI age groups. Similarly, smoking was not included as a covariate because it was queried only in those aged 12 years and above. To address the potential impact of omitting these covariates, separate analyses of nutrient intakes by breakfast group were conducted with and without their inclusion in children aged 6 to 11 and 12 to 17 years. We also examined whether observed differences in daily nutrient intakes between the RTEC-breakfast and other-breakfast groups were primarily due to the differences in intakes at breakfast, or were associated with the differences in dietary intakes during the rest of the day. To do this, nutrient intakes at breakfast and during the rest of the day (excluding breakfast) were compared between these two groups. Finally, the prevalence of nutrient inadequacy and the potential risk of excessive intakes were compared among the three breakfast groups using a $Z$-test and $P<0.05$ (Bonferroni-adjusted $P<0.0167$ ) to assess significance.

\section{Results}

\section{Participant characteristics}

Nationally weighted demographic characteristics are shown in Table 1 for the group as a whole and for each of the three breakfast groups. Breakfast non-consumers were the eldest of the three groups, and were less likely to use supplements than both groups of breakfast consumers. Among those aged 12 years and above, breakfast non-consumers had higher proportions classified as physically inactive and a higher average daily 'screen time' than breakfast consumers. They were also the most likely to smoke. Those consuming RTEC breakfasts were the youngest of the three groups and were more likely to be male than those consuming other types of breakfasts. Among those aged 12 years and above, RTEC-breakfast consumers had the lowest proportion who smoked and the highest proportion classified as physically active. Physical activity was also higher among RTEC-breakfast consumers aged 6 to 11 years compared with the other two groups. Finally, a smaller proportion of those consuming other types of breakfasts spoke English at home, compared with the other two groups. The proportions of consumers who were white, food secure and lived in an urban setting did not differ by breakfast group.

Overall, $10 \%$ of Canadian children did not eat breakfast, $33 \%$ consumed a breakfast that included RTEC and $57 \%$ consumed other types of breakfasts (Fig. 1). The proportion of those who skipped breakfast increased progressively across the age groups from $2 \%(4-8$ years) to $9 \%(9-13$ years) to $18 \%$ (14-18 years) $(P<0 \cdot 001)$, whereas the corresponding proportions of those consuming RTEC breakfasts decreased $(P<0.001)$. There were no changes with age in the proportion of those who consumed other types of breakfasts.

\section{Nutrient intakes}

The mean daily nutrient energy intake was lower in breakfast non-consumers than in both groups of breakfast consumers 
Table 1. Demographic characteristics of Canadian children and adolescents aged 4-18 years by breakfast group* (Mean values, standard errors and percentages)

\begin{tabular}{|c|c|c|c|c|c|c|c|c|}
\hline \multirow{2}{*}{ Characteristics } & \multicolumn{2}{|c|}{ All $(n$ 12281) } & \multicolumn{2}{|c|}{$\begin{array}{l}\text { No breakfast } \\
\quad(n 1471)\end{array}$} & \multicolumn{2}{|c|}{$\begin{array}{l}\text { Other breakfast } \\
\quad(n 6920)\end{array}$} & \multicolumn{2}{|c|}{$\begin{array}{c}\text { RTEC breakfast } \\
\text { ( } n \text { 3890) }\end{array}$} \\
\hline & $\%$ & SEM & $\%$ & SEM & $\%$ & SEM & $\%$ & SEM \\
\hline \multicolumn{9}{|l|}{ Age (years) } \\
\hline Mean & \multirow{2}{*}{\multicolumn{2}{|c|}{$\begin{array}{c}11 \cdot 2 \\
0.1\end{array}$}} & \multicolumn{2}{|c|}{$14.0^{\mathrm{a}}$} & \multicolumn{2}{|c|}{$11 \cdot 2^{\mathrm{b}}$} & \multicolumn{2}{|c|}{$10 \cdot 4^{c}$} \\
\hline SEM & & & \multicolumn{2}{|c|}{0.2} & \multicolumn{2}{|c|}{0.1} & \multicolumn{2}{|c|}{0.1} \\
\hline Male (\%) & 51.5 & 0.7 & $52 \cdot 0^{\mathrm{a}, \mathrm{b}}$ & $2 \cdot 3$ & $48 \cdot 7^{\mathrm{a}}$ & 1.0 & $56 \cdot 2^{\mathrm{b}}$ & $1 \cdot 3$ \\
\hline Physical activity (h/week), age $6-11$ years & \multirow{2}{*}{\multicolumn{2}{|c|}{$12 \cdot 8$}} & \multirow{2}{*}{\multicolumn{2}{|c|}{$11 \cdot 0^{\mathrm{a}}$}} & \multirow{2}{*}{\multicolumn{2}{|c|}{$12 \cdot 6^{\mathrm{a}}$}} & \multirow{2}{*}{\multicolumn{2}{|c|}{$13 \cdot 4^{\mathrm{b}}$}} \\
\hline Mean & & & & & & & & \\
\hline SEM & \multicolumn{2}{|c|}{0.2} & \multicolumn{2}{|c|}{0.6} & \multicolumn{2}{|c|}{0.2} & \multicolumn{2}{|c|}{0.2} \\
\hline \multicolumn{9}{|c|}{ Physical activity category (\%), age $12-18$ years $\dagger$} \\
\hline Active & $41 \cdot 0$ & $1 \cdot 0$ & $35 \cdot 7^{\mathrm{a}}$ & $2 \cdot 4$ & $40 \cdot 0^{\mathrm{a}}$ & 1.4 & $46 \cdot 0^{\mathrm{b}}$ & 1.9 \\
\hline Moderate & $25 \cdot 6$ & 0.9 & $23 \cdot 8$ & $2 \cdot 1$ & $26 \cdot 6$ & $1 \cdot 2$ & 24.4 & $1 \cdot 7$ \\
\hline Inactive & 33.4 & 1.0 & $40 \cdot 5^{\mathrm{a}}$ & $2 \cdot 4$ & $33 \cdot 4^{\mathrm{b}}$ & 1.4 & $29 \cdot 6^{\mathrm{b}}$ & 1.7 \\
\hline \multicolumn{9}{|l|}{ 'Screen time' $(\mathrm{h} / \mathrm{d})$, age $6-11$ years $\ddagger$} \\
\hline Mean & \multicolumn{2}{|c|}{$2 \cdot 6$} & \multicolumn{2}{|c|}{$2 \cdot 7$} & \multicolumn{2}{|c|}{$2 \cdot 6$} & \multicolumn{2}{|c|}{$2 \cdot 5$} \\
\hline SEM & \multicolumn{2}{|c|}{0.05} & \multicolumn{2}{|c|}{0.2} & \multicolumn{2}{|c|}{0.1} & \multicolumn{2}{|c|}{$0 \cdot 1$} \\
\hline 'Screen time' $(\mathrm{h} / \mathrm{d})$, age $12-17$ years $\ddagger$ & & & & & & & & \\
\hline Mean & & & & & & & & \\
\hline SEM & & & & & & & & \\
\hline Dietary supplement use (\% yes) & $34 \cdot 2$ & 0.7 & $26 \cdot 0^{\mathrm{a}}$ & $2 \cdot 0$ & $34 \cdot 2^{\mathrm{b}}$ & 1.0 & $36 \cdot 6^{\mathrm{b}}$ & $1 \cdot 3$ \\
\hline Food Secure (\% yes)§ & 91.2 & 0.4 & $90 \cdot 0$ & $1 \cdot 2$ & $92 \cdot 0$ & 0.6 & $90 \cdot 2$ & 0.8 \\
\hline Urban (\%) & $81 \cdot 3$ & 0.7 & 82.5 & $1 \cdot 7$ & $81 \cdot 0$ & 0.8 & $81 \cdot 3$ & $1 \cdot 2$ \\
\hline Smoking (\% smokers), age $12-18$ years & $6 \cdot 8$ & 0.5 & $14.9^{\mathrm{a}}$ & $2 \cdot 0$ & $6.4^{\mathrm{b}}$ & 0.7 & $2 \cdot 9^{\mathrm{c}}$ & 0.4 \\
\hline White (\%) & $80 \cdot 0$ & 0.7 & $80 \cdot 1$ & 1.9 & $80 \cdot 1$ & 0.9 & 79.9 & $1 \cdot 2$ \\
\hline English spoken at home (\%) & $70 \cdot 0$ & 0.7 & $73 \cdot 5^{\mathrm{a}}$ & 2.4 & $66 \cdot 5^{\mathrm{b}}$ & 1.0 & $75 \cdot 2^{\mathrm{a}}$ & 1.2 \\
\hline
\end{tabular}

RTEC, ready-to-eat cereal included with breakfast.

a,b,c Mean values within a row with unlike superscript letters were significantly different $(P<0.05$; Bonferroni-adjusted $P<0.0167)$.

* Data were obtained from the Canadian Community Health Survey, Cycle 2.2.

†Different questions were used to assess physical activity in children aged 6-11 v. 12-18 years. Physical activity was not assessed in children under the age of 6 years.

$\ddagger$ Different questions were used to assess 'screen time' (time spent on computers, playing video games and watching television) in children aged $6-11 \mathrm{v}$. $12-17$ years. Screen time was not assessed in those aged $<6$ or $\geq 18$ years.

$\S$ For respondents aged $\leq 17$ years, a knowledgeable adult member of the household was asked about the food security questions. Those aged 18 years responded on their own behalf.

(Table 2). With regard to nutrients, several patterns of differences in energy-adjusted intakes were observed for the group as a whole: (1) stepwise significant increases in the intakes of carbohydrate, fibre, thiamin, vitamin $\mathrm{B}_{6}$, vitamin $\mathrm{D}, \mathrm{Ca}, \mathrm{Fe}, \mathrm{Mg}, \mathrm{P}$ and $\mathrm{K}$ among breakfast non-consumers, other-breakfast consumers and RTEC-breakfast consumers; (2) stepwise significant decreases in the intakes of total fat, saturated fat and monounsaturated fat across the three groups; (3) significantly higher intakes of vitamin A, folate and vitamin $\mathrm{C}$ in both groups of breakfast consumers compared with non-consumers; (4) significantly lower intakes of polyunsaturated fat and cholesterol, and significantly higher intakes of sugars and riboflavin in RTEC-breakfast consumers compared with the other two groups; and (5) a significantly higher intake of niacin in other-breakfast consumers compared with breakfast non-consumers, neither of which differed significantly from RTEC-breakfast consumers. Intakes of protein, vitamin $\mathrm{B}_{12}, \mathrm{Zn}$ and $\mathrm{Na}$ did not differ among the breakfast groups. In general, similar patterns of differences were observed among males and females when examined separately (Table 2), as well as among those aged 4-8, 9-13 and 14-18 years (data not shown), although the differences were not always significant.

Analyses similar to those reported in Table 2 were also conducted separately for children aged 6 to 11 and 12 to 17 years to examine the potential impact of adding covariates that could not be included in the primary analysis: physical activity; 'screen time'; and smoking (12-17 years only). Of the 156 comparisons (two age groups, the twenty-six nutrients

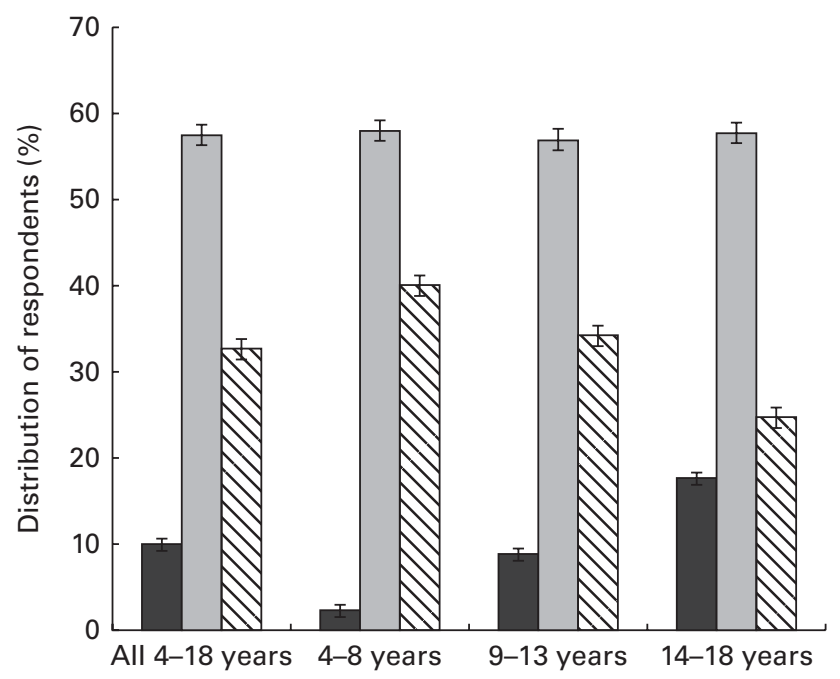

Fig. 1. Distribution of respondents by breakfast group and age. $\square$, No breakfast; $\square$, other types of breakfast; $\nabla$, breakfast that included ready-to-eat cereal. Data were obtained from the Canadian Community Health Survey Cycle 2.2. 
Table 2. Nutrient intakes of Canadian children and adolescents aged $4-18$ years by breakfast group ${ }^{*}$

(Weighted means with their standard errors)

\begin{tabular}{|c|c|c|c|c|c|c|c|c|c|c|c|c|c|c|c|c|c|c|}
\hline \multirow[b]{3}{*}{ Nutrients } & \multicolumn{6}{|c|}{ All breakfast group } & \multicolumn{6}{|c|}{ Male breakfast group } & \multicolumn{6}{|c|}{ Female breakfast group } \\
\hline & \multicolumn{2}{|c|}{ None $(n 1471)$} & \multicolumn{2}{|c|}{ Other $(n 6917)$} & \multicolumn{2}{|c|}{ RTEC ( $n$ 3890) } & \multicolumn{2}{|c|}{ None $(n 731)$} & \multicolumn{2}{|c|}{ Other ( $n$ 3335) } & \multicolumn{2}{|c|}{ RTEC (n 2191) } & \multicolumn{2}{|c|}{ None $(n 740)$} & \multicolumn{2}{|c|}{ Other ( $n$ 3582) } & \multicolumn{2}{|c|}{ RTEC ( $n$ 1699) } \\
\hline & Mean & SEM & Mean & SEM & Mean & SEM & Mean & SEM & Mean & SEM & Mean & SEM & Mean & SEM & Mean & SEM & Mean & SEM \\
\hline Energy (kJ) & $7736^{\mathrm{a}}$ & 188 & $9380^{\mathrm{b}}$ & 75 & $9619^{b}$ & 105 & $8221^{a}$ & 266 & $10401^{\mathrm{b}}$ & 29 & $2535^{\mathrm{b}}$ & 121 & $7209^{a}$ & 222 & $8355^{\mathrm{b}}$ & 84 & $8468^{b}$ & 134 \\
\hline Protein $(\mathrm{g})$ & $80 \cdot 0$ & $1 \cdot 3$ & $81 \cdot 2$ & 0.5 & $80 \cdot 8$ & 0.8 & $92 \cdot 0$ & $2 \cdot 0$ & $90 \cdot 7$ & 0.8 & $91 \cdot 2$ & 1.3 & $67 \cdot 1^{\mathrm{a}}$ & $1 \cdot 6$ & $71 \cdot 2^{b}$ & 0.6 & $69 \cdot 4^{a, b}$ & 0.8 \\
\hline Fat $(\mathrm{g})$ & $84 \cdot 3^{\mathrm{a}}$ & $1 \cdot 1$ & $80 \cdot 3^{b}$ & 0.4 & $73 \cdot 4^{\mathrm{c}}$ & 0.5 & $91 \cdot 6^{\mathrm{a}}$ & 1.4 & $89 \cdot 0^{\mathrm{a}}$ & 0.6 & $82 \cdot 2^{\mathrm{b}}$ & 0.8 & $76 \cdot 5^{\mathrm{a}}$ & 1.6 & $71 \cdot 2^{\mathrm{b}}$ & 0.5 & $64 \cdot 1^{\mathrm{c}}$ & 0.7 \\
\hline SFA (g) & $29 \cdot 1^{a}$ & 0.4 & $27 \cdot 8^{\mathrm{b}}$ & 0.2 & $26 \cdot 3^{c}$ & 0.3 & $31 \cdot 8^{\mathrm{a}}$ & 0.6 & $30 \cdot 8^{a}$ & 0.3 & $29 \cdot 4^{\mathrm{b}}$ & 0.4 & $26 \cdot 2^{\mathrm{a}}$ & 0.7 & $24 \cdot 6^{a}$ & 0.3 & $22 \cdot 9^{\mathrm{b}}$ & 0.4 \\
\hline MUFA (g) & $33 \cdot 6^{a}$ & 0.6 & $31.5^{\mathrm{b}}$ & 0.2 & $28 \cdot 0^{\mathrm{C}}$ & 0.2 & $36 \cdot 4^{\mathrm{a}}$ & 0.7 & $35 \cdot 1^{\mathrm{a}}$ & 0.3 & $31.5^{\mathrm{b}}$ & 0.4 & $30.5^{a}$ & 1.0 & $27 \cdot 7^{\mathrm{b}}$ & 0.2 & $24 \cdot 2^{\mathrm{c}}$ & 0.3 \\
\hline PUFA (g) & $13 \cdot 6^{a}$ & 0.3 & $13 \cdot 2^{\mathrm{a}}$ & 0.1 & $11 \cdot 8^{\mathrm{b}}$ & 0.2 & $14 \cdot 5^{a}$ & 0.4 & $14.5^{\mathrm{a}}$ & 0.2 & $13 \cdot 1^{\mathrm{b}}$ & 0.2 & $12 \cdot 5^{\mathrm{a}}$ & 0.6 & $11 \cdot 8^{\mathrm{a}}$ & 0.2 & $10 \cdot 5^{\mathrm{b}}$ & 0.2 \\
\hline Cholesterol (mg) & $257^{\mathrm{a}}$ & 9 & $256^{\mathrm{a}}$ & 3 & $207^{\mathrm{b}}$ & 3 & $305^{\mathrm{a}}$ & 14 & $292^{\mathrm{a}}$ & 5 & $238^{\mathrm{b}}$ & 5 & $207^{\mathrm{a}}$ & 10 & $218^{\mathrm{a}}$ & 4 & $173^{\mathrm{b}}$ & 4 \\
\hline Carbohydrate $(\mathrm{g})$ & $288^{\mathrm{a}}$ & 3 & $299^{b}$ & 1 & $317^{\mathrm{c}}$ & 1 & $316^{a}$ & 4 & $327^{a}$ & 2 & $344^{b}$ & 2 & $260^{\mathrm{a}}$ & 4 & $270^{\mathrm{b}}$ & 1 & $288^{c}$ & 2 \\
\hline Sugars $(g)$ & $138^{\mathrm{a}}$ & 2 & $135^{\mathrm{a}}$ & 1 & $145^{\mathrm{b}}$ & 2 & $150^{a, b}$ & 4 & $149^{\mathrm{a}}$ & 2 & $158^{\mathrm{b}}$ & 2 & $125^{a, b}$ & 3 & $120^{\mathrm{a}}$ & 1 & $131^{b}$ & 2 \\
\hline Fibre $(\mathrm{g})$ & $13 \cdot 2^{\mathrm{a}}$ & 0.3 & $14 \cdot 9^{\mathrm{b}}$ & 0.1 & $16 \cdot 1^{\mathrm{c}}$ & 0.2 & $14 \cdot 0^{\mathrm{a}}$ & 0.4 & $15 \cdot 7^{\mathrm{b}}$ & 0.2 & $17 \cdot 4^{\mathrm{c}}$ & 0.3 & $12 \cdot 3^{\mathrm{a}}$ & 0.4 & $14 \cdot 0^{\mathrm{b}}$ & 0.2 & $14 \cdot 8^{\mathrm{c}}$ & 0.3 \\
\hline Vitamin A ( $\mu \mathrm{g}$ RAE) & $560^{a}$ & 19 & $647^{b}$ & 9 & $644^{\mathrm{b}}$ & 11 & $581^{a}$ & 30 & $692^{b}$ & 14 & $699^{\mathrm{b}}$ & 15 & 537 & 26 & 599 & 11 & 584 & 15 \\
\hline Thiamin (mg) & $1.5^{\mathrm{a}}$ & 0.02 & $1.7^{\mathrm{b}}$ & 0.01 & $2 \cdot 2^{c}$ & 0.03 & $1 \cdot 6^{\mathrm{a}}$ & 0.04 & $1.8^{\mathrm{b}}$ & 0.02 & $2 \cdot 5^{\mathrm{c}}$ & 0.04 & $1.4^{\mathrm{a}}$ & 0.03 & $1.5^{\mathrm{b}}$ & 0.01 & $1.9^{\mathrm{c}}$ & 0.04 \\
\hline Riboflavin (mg) & $2 \cdot 0^{\mathrm{a}}$ & 0.06 & $2 \cdot 1^{\mathrm{a}}$ & 0.01 & $2 \cdot 3^{\mathrm{b}}$ & 0.03 & $2 \cdot 3^{\mathrm{a}}$ & 0.08 & $2 \cdot 3^{\mathrm{a}}$ & 0.02 & $2 \cdot 6^{\mathrm{b}}$ & 0.05 & $1 \cdot 8^{\mathrm{a}, \mathrm{b}}$ & 0.10 & $1.9^{\mathrm{a}}$ & 0.02 & $2 \cdot 0^{\mathrm{b}}$ & 0.04 \\
\hline Niacin (mg NE) & $34.5^{\mathrm{a}}$ & 0.6 & $36 \cdot 0^{\mathrm{b}}$ & 0.2 & $36 \cdot 0^{a, b}$ & 0.4 & $39 \cdot 2$ & 0.9 & $40 \cdot 0$ & 0.4 & $40 \cdot 2$ & 0.6 & $29 \cdot 5^{\mathrm{a}}$ & 0.7 & $31.9^{\mathrm{b}}$ & 0.3 & $31 \cdot 3^{\mathrm{b}}$ & 0.4 \\
\hline Vitamin $B_{6}(\mathrm{mg})$ & $1.5^{\mathrm{a}}$ & 0.03 & $1 \cdot 6^{\mathrm{b}}$ & 0.01 & $1.8^{\mathrm{C}}$ & 0.02 & $1 \cdot 7^{\mathrm{a}}$ & 0.05 & $1.8^{\mathrm{a}}$ & 0.02 & $2 \cdot 0^{\mathrm{b}}$ & 0.03 & $1 \cdot 3^{\mathrm{a}}$ & 0.04 & $1.5^{\mathrm{b}}$ & 0.02 & $1.6^{\mathrm{c}}$ & 0.03 \\
\hline Folate (DFE) & $404^{\mathrm{a}}$ & 8 & $462^{b}$ & 4 & $458^{\mathrm{b}}$ & 5 & $435^{\mathrm{a}}$ & 12 & $498^{\mathrm{b}}$ & 6 & $492^{\mathrm{b}}$ & 7 & $373^{\mathrm{a}}$ & 10 & $424^{\mathrm{b}}$ & 5 & $424^{\mathrm{b}}$ & 7 \\
\hline Vitamin $B_{12}(\mu \mathrm{g})$ & $4 \cdot 1$ & 0.2 & 4.0 & 0.1 & $4 \cdot 1$ & 0.1 & $5 \cdot 0$ & 0.4 & 4.6 & 0.1 & 4.7 & 0.2 & $3.0^{\mathrm{a}}$ & 0.1 & $3.5^{\mathrm{b}}$ & 0.1 & $3 \cdot 4^{a, b}$ & 0.1 \\
\hline Vitamin C (mg) & $129^{a}$ & 6 & $156^{b}$ & 2 & $148^{\mathrm{b}}$ & 3 & $129^{a}$ & 7 & $162^{\mathrm{b}}$ & 4 & $156^{b}$ & 4 & 130 & 9 & 148 & 3 & 140 & 4 \\
\hline Vitamin D $(\mu \mathrm{g})$ & $5 \cdot 3^{\mathrm{a}}$ & 0.2 & $6 \cdot 0^{\mathrm{b}}$ & 0.1 & $6 \cdot 9^{c}$ & 0.1 & $6 \cdot 1^{\mathrm{a}}$ & 0.2 & $6 \cdot 8^{\mathrm{b}}$ & 0.1 & $7 \cdot 6^{\mathrm{c}}$ & 0.2 & $4 \cdot 5^{\mathrm{a}}$ & 0.3 & $5 \cdot 2^{b}$ & 0.1 & $6 \cdot 1^{\mathrm{c}}$ & 0.2 \\
\hline $\mathrm{Ca}(\mathrm{mg})$ & $974^{\mathrm{a}}$ & 24 & $1042^{b}$ & 9 & $1192^{c}$ & 15 & $1075^{\mathrm{a}}$ & 35 & $1148^{\mathrm{a}}$ & 15 & $1310^{\mathrm{b}}$ & 19 & $872^{\mathrm{a}}$ & 31 & $928^{\mathrm{a}}$ & 12 & $1063^{b}$ & 24 \\
\hline $\mathrm{Fe}(\mathrm{mg})$ & $12 \cdot 4^{\mathrm{a}}$ & 0.2 & $13 \cdot 1^{\mathrm{b}}$ & 0.1 & $18 \cdot 2^{\mathrm{C}}$ & 0.2 & $13 \cdot 8^{\mathrm{a}}$ & 0.3 & $14 \cdot 3^{\mathrm{a}}$ & 0.1 & $20 \cdot 0^{\mathrm{b}}$ & 0.2 & $10 \cdot 8^{\mathrm{a}}$ & 0.2 & $11 \cdot 8^{\mathrm{b}}$ & 0.1 & $16 \cdot 1^{\mathrm{c}}$ & 0.3 \\
\hline$M g(\mathrm{mg})$ & $257^{\mathrm{a}}$ & 3 & $285^{\mathrm{b}}$ & 2 & $306^{\mathrm{c}}$ & 2 & $283^{\mathrm{a}}$ & 5 & $308^{\mathrm{a}}$ & 2 & $335^{\mathrm{C}}$ & 3 & $230^{\mathrm{a}}$ & 4 & $260^{\mathrm{b}}$ & 2 & $275^{\mathrm{c}}$ & 3 \\
\hline $\mathrm{P}(\mathrm{mg})$ & $1301^{\mathrm{a}}$ & 18 & $1371^{\mathrm{b}}$ & 8 & $1463^{c}$ & 12 & $1472^{\mathrm{a}}$ & 26 & $1519^{a}$ & 12 & $1626^{\mathrm{b}}$ & 16 & $1121^{\mathrm{a}}$ & 24 & $1215^{b}$ & 10 & $1285^{c}$ & 17 \\
\hline $\mathrm{Zn}(\mathrm{mg})$ & $10 \cdot 8$ & 0.2 & 10.9 & 0.1 & $11 \cdot 0$ & 0.1 & $12 \cdot 4$ & 0.3 & $12 \cdot 1$ & 0.2 & $12 \cdot 4$ & 0.2 & $9 \cdot 0^{\mathrm{a}}$ & 0.2 & $9 \cdot 6^{\mathrm{b}}$ & 0.2 & $9 \cdot 4^{\mathrm{a}, \mathrm{b}}$ & 0.1 \\
\hline $\mathrm{Na}(\mathrm{mg})$ & 3187 & 59 & 3204 & 24 & 3125 & 31 & 3486 & 80 & 3513 & 35 & 3463 & 45 & 2864 & 87 & 2877 & 31 & 2761 & 37 \\
\hline $\mathrm{K}(\mathrm{mg})$ & $2647^{a}$ & 43 & $2889^{b}$ & 18 & $2991^{c}$ & 26 & $2915^{\mathrm{a}}$ & 60 & $3123^{b}$ & 27 & $3290^{c}$ & 37 & $2367^{a}$ & 61 & $2638^{b}$ & 24 & $2664^{\mathrm{b}}$ & 32 \\
\hline
\end{tabular}

RTEC, ready-to-eat cereal included with breakfast; RAE, retinol activity equivalents; NE, niacin equivalents; DFE, dietary folate equivalents.

${ }^{a, b, c}$ Mean values within a row with unlike superscript letters were significantly different $(P<0.05$; Bonferroni-adjusted $P<0.0167)$.

"Data were obtained from the Canadian Community Health Survey, Cycle 2.2, adjusted for energy (for nutrients), age, sex, race, dietary supplement use, food security and language spoken at home. 
included in Table 2 and three breakfast group comparisons for each nutrient in each age group), 152 similar conclusions about breakfast group differences were reached with and without the added covariates (data not shown). For two nutrients (total fat in 12- to 17-year-olds and monounsaturated fat in 6- to 11-year-olds), one of the three breakfast group differences shifted from significant to not significant, and for two other nutrients (riboflavin and niacin in 12- to 17-year-olds) one of the three breakfast group differences shifted from not significant to significant.

To assess whether the differences in dietary intakes between the RTEC-breakfast and other-breakfast groups were associated with intake at breakfast per se, or whether they reflected differences in nutrient intake throughout the day, we compared the intakes of these two groups at the breakfast meal and during the rest of the day (Table 3). Breakfast intakes of energy, protein, niacin and folate did not differ between the groups. However, all the other nutrients differed. The RTEC-breakfast group had higher breakfast intakes of carbohydrate, sugars, fibre, vitamin $\mathrm{D}$, thiamin, riboflavin, vitamin $\mathrm{B}_{6}, \mathrm{Ca}, \mathrm{P}, \mathrm{Mg}, \mathrm{Fe}, \mathrm{Zn}$ and $\mathrm{K}$ (all $P<0.0001$ ), as well as vitamin $\mathrm{B}_{12}(P<0 \cdot 01)$. The other-breakfast consumers had higher intakes of total fat, saturated fat, monounsaturated fat, polyunsaturated fat, cholesterol and Na (all $P<0 \cdot 001$ ), as well as vitamins A and $\mathrm{C}(P<0 \cdot 01)$. During the rest of the day, intakes of energy, macronutrients and most micronutrients were similar between the groups, although riboflavin was slightly higher in the RTEC-breakfast group and $\mathrm{Zn}$ was slightly higher in the other-breakfast group (both $P<0 \cdot 05$ ).

\section{Nutrient adequacy}

Table 4 shows the prevalence of nutrient inadequacy from food sources alone and from the combination of food sources plus supplements (total intakes) for nutrients with an EAR. There was considerable variability across the nutrients in the prevalence of inadequacy, ranging from close to zero for niacin and riboflavin, to considerably higher proportions for $\mathrm{Ca}, \mathrm{Mg}$, vitamin A and vitamin D. Variability was also observed by breakfast group. For food and total intakes of vitamin D, $\mathrm{Ca}, \mathrm{Fe}$ and $\mathrm{Mg}$, the prevalence of inadequacy was significantly greater in breakfast non-consumers than in other-breakfast consumers, who in turn had a significantly greater prevalence of inadequacy than RTEC-breakfast consumers. For food and total intakes of vitamin $\mathrm{A}, \mathrm{P}$ and $\mathrm{Zn}$, and for total intakes of vitamin $\mathrm{C}$, the prevalence of inadequacy was significantly greater among the breakfast non-consumers than among the groups of breakfast consumers, which did not differ. For food and total intakes of vitamin $\mathrm{B}_{6}$ and for food intakes of folate, the prevalence of inadequacy was significantly greater

Table 3. Nutrient intake of Canadian children and adolescents consuming breakfasts with and without ready-to-eat cereal (RTEC) at the breakfast meal and during the rest of the dayt

(Weighted means with their standard errors)

\begin{tabular}{|c|c|c|c|c|c|c|c|c|}
\hline \multirow[b]{3}{*}{ Nutrients } & \multicolumn{4}{|c|}{ Intake at breakfast } & \multicolumn{4}{|c|}{ Intake during the rest of the day } \\
\hline & \multicolumn{2}{|c|}{$\begin{array}{l}\text { Other breakfast } \\
\text { ( }(\text { 6917) }\end{array}$} & \multicolumn{2}{|c|}{$\begin{array}{l}\text { RTEC breakfast } \\
\text { (n 3890) }\end{array}$} & \multicolumn{2}{|c|}{$\begin{array}{l}\text { Other breakfast } \\
\text { (n 6917) }\end{array}$} & \multicolumn{2}{|c|}{$\begin{array}{l}\text { RTEC breakfast } \\
\text { ( }(n 3890)\end{array}$} \\
\hline & Mean & SEM & Mean & SEM & Mean & SEM & Mean & SEM \\
\hline Energy (kJ) & 1699 & 21 & 1761 & 25 & 7602 & 21 & 7540 & 25 \\
\hline Protein $(\mathrm{g})$ & $13 \cdot 7$ & 0.2 & $13 \cdot 9$ & 0.2 & $67 \cdot 6$ & 0.5 & $66 \cdot 9$ & 0.8 \\
\hline Fat $(\mathrm{g})$ & $14 \cdot 1$ & 0.3 & $8 \cdot 4^{\star \star \star}$ & 0.2 & $66 \cdot 2$ & 0.4 & $65 \cdot 1$ & 0.6 \\
\hline SFA (g) & $5 \cdot 1$ & 0.1 & $3 \cdot 7^{\star \star \star}$ & 0.1 & $22 \cdot 7$ & 0.2 & $22 \cdot 6$ & 0.3 \\
\hline MUFA (g) & $5 \cdot 1$ & 0.1 & $2 \cdot 4^{\star \star \star}$ & 0.1 & $26 \cdot 4$ & 0.2 & $25 \cdot 6$ & 0.3 \\
\hline PUFA (g) & $2 \cdot 3$ & 0.05 & $1 \cdot 1^{\star \star \star}$ & 0.04 & $10 \cdot 9$ & 0.1 & $10 \cdot 7$ & 0.2 \\
\hline Cholesterol (mg) & $72 \cdot 0$ & $2 \cdot 4$ & $23 \cdot 3^{\star \star \star}$ & $1 \cdot 1$ & $183 \cdot 9$ & 2.5 & $183 \cdot 7$ & $3 \cdot 4$ \\
\hline Carbohydrate (g) & $57 \cdot 6$ & 0.8 & $74 \cdot 3^{\star \star \star}$ & $1 \cdot 2$ & $241 \cdot 8$ & $1 \cdot 2$ & $242 \cdot 1$ & 1.6 \\
\hline Sugars (g) & $27 \cdot 4$ & 0.5 & $36 \cdot 7^{\star \star \star}$ & 0.7 & $108 \cdot 2$ & 1.0 & $108 \cdot 0$ & 1.5 \\
\hline Fibre (g) & $2 \cdot 5$ & 0.05 & $3 \cdot 6^{\star \star \star}$ & 0.1 & $12 \cdot 3$ & 0.1 & $12 \cdot 5$ & 0.2 \\
\hline Vitamin A ( $\mu$ g RAE) & $154 \cdot 2$ & $3 \cdot 1$ & $140 \cdot 3^{\star \star}$ & $3 \cdot 1$ & $494 \cdot 8$ & $8 \cdot 2$ & $502 \cdot 9$ & $10 \cdot 2$ \\
\hline Thiamin (mg) & 0.37 & 0.01 & $0.87^{\star \star \star}$ & 0.02 & 1.32 & 0.01 & $1 \cdot 33$ & 0.02 \\
\hline Riboflavin (mg) & 0.52 & 0.01 & $0.65^{\star \star \star}$ & 0.02 & 1.60 & 0.01 & $1.67^{*}$ & 0.03 \\
\hline Niacin (mg NE) & $5 \cdot 8$ & 0.1 & $6 \cdot 1$ & 0.1 & $30 \cdot 2$ & 0.2 & 29.9 & 0.4 \\
\hline Vitamin $B_{6}(\mathrm{mg})$ & 0.29 & 0.01 & $0.50^{\star \star \star}$ & 0.01 & 1.34 & 0.01 & 1.35 & 0.02 \\
\hline Folate (DFE) & 93.9 & 1.5 & $92 \cdot 8$ & $2 \cdot 0$ & $372 \cdot 8$ & 3.7 & $368 \cdot 3$ & 4.4 \\
\hline Vitamin $B_{12}(\mu \mathrm{g})$ & 0.84 & 0.02 & $0.92^{\star \star}$ & 0.02 & $3 \cdot 2$ & 0.1 & $3 \cdot 2$ & 0.1 \\
\hline Vitamin C (mg) & $35 \cdot 2$ & $1 \cdot 1$ & $29 \cdot 6^{\star \star}$ & 1.5 & $120 \cdot 4$ & $2 \cdot 1$ & $117 \cdot 6$ & $2 \cdot 6$ \\
\hline Vitamin D $(\mu \mathrm{g})$ & $1 \cdot 8$ & 0.04 & $2 \cdot 5^{\star \star \star}$ & 0.05 & $4 \cdot 2$ & 0.1 & $4 \cdot 4$ & 0.1 \\
\hline $\mathrm{Ca}(\mathrm{mg})$ & 258 & 5 & $277^{\star \star \star}$ & 7 & 786 & 8 & 814 & 13 \\
\hline $\mathrm{Fe}(\mathrm{mg})$ & 2.5 & 0.05 & $7 \cdot 4^{\star * *}$ & 0.2 & $10 \cdot 6$ & 0.1 & $10 \cdot 7$ & 0.1 \\
\hline $\mathrm{Mg}(\mathrm{mg})$ & $59 \cdot 5$ & 0.9 & $78 \cdot 6^{\star \star \star}$ & 1.4 & $225 \cdot 6$ & 1.4 & $227 \cdot 2$ & 1.9 \\
\hline $\mathrm{Zn}(\mathrm{mg})$ & $1 \cdot 8$ & 0.03 & $2 \cdot 2^{\star \star \star}$ & 0.04 & $9 \cdot 1$ & 0.1 & $8 \cdot 8^{*}$ & 0.1 \\
\hline $\mathrm{Na}(\mathrm{mg})$ & 624 & 15 & $553^{\star \star *}$ & 12 & 2658 & 24 & 2620 & 30 \\
\hline $\mathrm{K}(\mathrm{mg})$ & 573 & 8 & $670^{\star * *}$ & 11 & 2317 & 16 & 2318 & 23 \\
\hline
\end{tabular}

RAE, retinol activity equivalents; NE, niacin equivalents; DFE, dietary folate equivalents.

Mean value was significantly different from that of the other-breakfast group: ${ }^{*} P<0.05,{ }^{* \star} P<0.01,{ }^{\star * \star} P<0.0001$.

† Data were obtained from the Canadian Community Health Survey Cycle 2.2. 


\section{Nes British Journal of Nutrition}

Table 4. Prevalence of inadequate vitamin and mineral intakes (for nutrients with an estimated average requirement (EAR)) by breakfast group in Canadian children aged 4-18 years ${ }^{\star}$ (Mean values for percentage below the EAR with their standard errors)

\begin{tabular}{|c|c|c|c|c|c|c|c|c|c|c|c|c|c|c|c|c|c|c|}
\hline \multirow[b]{3}{*}{ Nutrients } & \multicolumn{6}{|c|}{ All breakfast group } & \multicolumn{6}{|c|}{ Male breakfast group } & \multicolumn{6}{|c|}{ Female breakfast group } \\
\hline & \multicolumn{2}{|c|}{$\begin{array}{c}\text { None } \\
(n 1471)\end{array}$} & \multicolumn{2}{|c|}{$\begin{array}{c}\text { Other } \\
(n \text { 6917) }\end{array}$} & \multicolumn{2}{|c|}{$\begin{array}{c}\text { RTEC } \\
(n 3890)\end{array}$} & \multicolumn{2}{|c|}{$\begin{array}{l}\text { None } \\
(n 731)\end{array}$} & \multicolumn{2}{|c|}{$\begin{array}{c}\text { Other } \\
(n 3335)\end{array}$} & \multicolumn{2}{|c|}{$\begin{array}{l}\text { RTEC } \\
(n 2191)\end{array}$} & \multicolumn{2}{|c|}{$\begin{array}{l}\text { None } \\
(n 740)\end{array}$} & \multicolumn{2}{|c|}{$\begin{array}{c}\text { Other } \\
(n \text { 3582) }\end{array}$} & \multicolumn{2}{|c|}{$\begin{array}{l}\text { RTEC } \\
(n \text { 1699) }\end{array}$} \\
\hline & Mean & SEM & Mean & SEM & Mean & SEM & Mean & SEM & Mean & SEM & Mean & SEM & Mean & SEM & Mean & SEM & Mean & SEM \\
\hline \multicolumn{19}{|l|}{ Vitamin A } \\
\hline Food & $50 \cdot 0^{\mathrm{a}}$ & $5 \cdot 2$ & $21.6^{\mathrm{b}}$ & $2 \cdot 4$ & $17 \cdot 8^{\mathrm{b}}$ & 1.5 & $49 \cdot 1^{a}$ & $7 \cdot 4$ & $18 \cdot 7^{\mathrm{b}}$ & $2 \cdot 2$ & $18 \cdot 2^{\mathrm{b}}$ & $2 \cdot 8$ & $51 \cdot 1^{\mathrm{a}}$ & 5.9 & $24 \cdot 2^{b}$ & 3.4 & $17 \cdot 3^{\mathrm{b}}$ & 3.3 \\
\hline Food and supplements & $45 \cdot 4^{\mathrm{a}}$ & $4 \cdot 7$ & $18 \cdot 1^{\mathrm{b}}$ & 1.9 & $14 \cdot 0^{\mathrm{b}}$ & 1.3 & $44.2^{\mathrm{a}}$ & 6.5 & $15 \cdot 7^{\mathrm{b}}$ & 1.9 & $13 \cdot 6^{\mathrm{b}}$ & $2 \cdot 0$ & $46.0^{\mathrm{a}}$ & $5 \cdot 3$ & $20 \cdot 5^{\mathrm{b}}$ & $2 \cdot 6$ & $14 \cdot 3^{\mathrm{b}}$ & 2.9 \\
\hline \multicolumn{19}{|l|}{ Thiamin } \\
\hline Food & $6 \cdot 1$ & 3.9 & 0.5 & 0.3 & 0.1 & 0.1 & $8 \cdot 2$ & 5.6 & 0.1 & 0.1 & 0.1 & 0.1 & 4.4 & 5.5 & 1.0 & 0.5 & 0.1 & 0.1 \\
\hline Food and supplements & $5 \cdot 6$ & 4.0 & 0.5 & 0.2 & 0.3 & 0.2 & 7.5 & $5 \cdot 2$ & 0.1 & 0.1 & 0.1 & 0.1 & 3.9 & $3 \cdot 6$ & 0.8 & 0.5 & 0.5 & 0.4 \\
\hline \multicolumn{19}{|l|}{ Riboflavin } \\
\hline Food & $1 \cdot 8$ & 1.5 & 0.2 & 0.1 & 0.1 & 0.1 & $2 \cdot 2$ & 1.1 & 0.1 & 0.1 & 0.1 & 0.1 & 1.6 & $2 \cdot 7$ & 0.5 & 0.2 & 0.1 & 0.1 \\
\hline Food and supplements & 1.7 & $1 \cdot 2$ & 0.3 & 0.1 & 0.3 & 0.2 & $2 \cdot 2$ & 0.9 & 0.1 & 0.1 & 0.1 & 0.1 & 1.4 & $2 \cdot 3$ & 0.4 & 0.2 & 0.5 & 0.4 \\
\hline \multicolumn{19}{|l|}{ Niacin } \\
\hline Food & 0.0 & 0.0 & 0.0 & 0.0 & 0.03 & 0.04 & 0.0 & 0.0 & 0.0 & 0.0 & 0.02 & 0.06 & 0.0 & 0.0 & 0.0 & 0.0 & 0.04 & 0.09 \\
\hline Food and supplements & 0.03 & 0.05 & 0.0 & 0.03 & 0.0 & 0.03 & 0.01 & 0.05 & 0.0 & 0.01 & 0.0 & 0.03 & 0.05 & 0.07 & 0.02 & 0.02 & 0.4 & 0.5 \\
\hline Vitamin $B_{6}$ & & & & & & & & & & & & & & & & & & \\
\hline Food & $10 \cdot 7^{\mathrm{a}}$ & $4 \cdot 0$ & $2 \cdot 8^{\mathrm{a}, \mathrm{b}}$ & 0.9 & $0.7^{b}$ & 0.4 & $3 \cdot 1$ & $2 \cdot 2$ & 0.9 & 0.4 & 0.4 & 0.4 & $19 \cdot 3^{\mathrm{a}}$ & $6 \cdot 8$ & $4 \cdot 5^{\mathrm{a}, \mathrm{b}}$ & 1.7 & $1 \cdot 1^{b}$ & 0.6 \\
\hline Food and supplements & $9 \cdot 5^{\mathrm{a}}$ & 3.3 & $2 \cdot 4^{\mathrm{a}, \mathrm{b}}$ & 0.8 & $0 \cdot 8^{\mathrm{b}}$ & 0.4 & $2 \cdot 6$ & $2 \cdot 3$ & 0.8 & 0.4 & 0.4 & 0.4 & $16 \cdot 6^{\mathrm{a}}$ & $6 \cdot 3$ & $4 \cdot 0^{\mathrm{a}, \mathrm{b}}$ & 1.4 & $1.4^{\mathrm{b}}$ & 0.8 \\
\hline Folate & & & & & & & & & & & & & & & & & & \\
\hline Food & $23.8^{\mathrm{a}}$ & 8.4 & $3.5^{\mathrm{a}, \mathrm{b}}$ & 1.2 & $2 \cdot 7^{\mathrm{b}}$ & 1.4 & $21 \cdot 0$ & $13 \cdot 0$ & $1 \cdot 1$ & 0.6 & 2.4 & 1.7 & $27 \cdot 9$ & $11 \cdot 8$ & $5 \cdot 7$ & $2 \cdot 0$ & $3 \cdot 3$ & 1.9 \\
\hline Food and supplements & $21 \cdot 3$ & $8 \cdot 2$ & $3 \cdot 1$ & 1.0 & 2.5 & 1.2 & 18.5 & $10 \cdot 4$ & 1.0 & 0.5 & $1 \cdot 8$ & 1.3 & $24 \cdot 3$ & $11 \cdot 2$ & $5 \cdot 1$ & 1.6 & 3.3 & 1.8 \\
\hline Vitamin $B_{12}$ & & & & & & & & & & & & & & & & & & \\
\hline Food & $7 \cdot 1$ & 5.9 & $2 \cdot 2$ & $1 \cdot 1$ & 1.5 & 0.5 & 0.03 & 1.5 & 1.0 & 0.7 & 0.3 & 0.3 & $14 \cdot 2$ & $12 \cdot 0$ & 3.4 & 1.7 & $2 \cdot 9$ & 1.0 \\
\hline Food and supplements & $6 \cdot 2$ & $5 \cdot 2$ & $2 \cdot 0$ & 0.9 & 1.4 & 0.5 & 0.03 & 0.8 & 0.9 & 0.5 & 0.2 & 0.2 & 12.6 & $10 \cdot 9$ & 3.0 & 1.5 & 2.7 & 0.9 \\
\hline Vitamin C & & & & & & & & & & & & & & & & & & \\
\hline Food & 11.8 & 4.5 & 3.3 & 0.6 & $3 \cdot 1$ & 1.0 & 4.5 & 3.9 & 4.1 & 0.9 & 4.5 & 1.7 & $19.0^{\mathrm{a}}$ & $5 \cdot 8$ & $2 \cdot 7^{\mathrm{b}}$ & 0.8 & $1.3^{\mathrm{b}}$ & 0.7 \\
\hline Food and supplements & $10 \cdot 6^{\mathrm{a}}$ & 2.5 & $3.0^{\mathrm{b}}$ & 0.4 & $2 \cdot 6^{b}$ & 0.8 & $5 \cdot 0$ & 3.7 & 3.6 & 0.7 & $3 \cdot 3$ & $1 \cdot 2$ & $16 \cdot 4^{\mathrm{a}}$ & $5 \cdot 1$ & $2 \cdot 2^{b}$ & 0.5 & $1.5^{\mathrm{b}}$ & 0.7 \\
\hline Vitamin D & & & & & & & & & & & & & & & & & & \\
\hline Food & $95 \cdot 7^{\mathrm{a}}$ & $2 \cdot 4$ & $88.5^{\mathrm{b}}$ & 1.3 & $81 \cdot 6^{\mathrm{c}}$ & 1.9 & $93.5^{\mathrm{a}}$ & 4.8 & $81 \cdot 1^{\mathrm{a}, \mathrm{b}}$ & $2 \cdot 1$ & $75 \cdot 9^{\mathrm{b}}$ & 4.4 & 98.6 & 2.4 & $95 \cdot 3$ & 1.3 & 88.7 & 3.6 \\
\hline Food and supplements & $85 \cdot 2^{a}$ & $2 \cdot 7$ & $70 \cdot 3^{b}$ & 1.3 & $61.9^{\mathrm{C}}$ & 1.9 & $82 \cdot 6^{\mathrm{a}}$ & $4 \cdot 3$ & $63 \cdot 7^{\mathrm{b}}$ & 1.8 & $56 \cdot 8^{\mathrm{b}}$ & 3.6 & $87 \cdot 9^{a}$ & $2 \cdot 3$ & $76.6^{\mathrm{b}}$ & 1.6 & $68 \cdot 6^{\mathrm{C}}$ & 2.9 \\
\hline $\mathrm{Ca}$ & & & & & & & & & & & & & & & & & & \\
\hline Food & $79.8^{\mathrm{a}}$ & 3.7 & $55 \cdot 1^{\mathrm{b}}$ & 2.5 & $33 \cdot 2^{\mathrm{c}}$ & 3.0 & $76 \cdot 7^{\mathrm{a}}$ & $6 \cdot 4$ & $42 \cdot 6^{\mathrm{b}}$ & 3.4 & $25 \cdot 8^{\mathrm{c}}$ & 3.6 & $83 \cdot 1^{\mathrm{a}}$ & $4 \cdot 3$ & $66 \cdot 9^{\mathrm{b}}$ & $2 \cdot 8$ & $42 \cdot 8^{\mathrm{c}}$ & $5 \cdot 2$ \\
\hline Food and supplements & $78 \cdot 1^{\mathrm{a}}$ & 3.9 & $51.9^{\mathrm{b}}$ & 2.5 & $30 \cdot 7^{\mathrm{c}}$ & $2 \cdot 8$ & $75 \cdot 4^{\mathrm{a}}$ & 5.7 & $40 \cdot 0^{\mathrm{b}}$ & 3.5 & $23 \cdot 6^{\mathrm{c}}$ & 3.4 & $81 \cdot 2^{\mathrm{a}}$ & $4 \cdot 1$ & $63 \cdot 3^{\mathrm{b}}$ & $2 \cdot 7$ & $40 \cdot 0^{c}$ & $4 . \overline{7}$ \\
\hline $\mathrm{Fe}$ & & & & & & & & & & & & & & & & & & \\
\hline Food & $9 \cdot 7^{\mathrm{a}}$ & $2 \cdot 6$ & $2.9^{\mathrm{b}}$ & 0.5 & $0.3^{c}$ & 0.1 & $1 \cdot 9^{\mathrm{a}, \mathrm{b}}$ & 1.4 & $0.7^{\mathrm{a}}$ & 0.2 & $0.1^{b}$ & 0.05 & $18 \cdot 1^{\mathrm{a}}$ & 4.9 & $5 \cdot 1^{\mathrm{b}}$ & 0.8 & $0.5^{\mathrm{c}}$ & 0.2 \\
\hline Food and supplements & $9 \cdot 1^{\mathrm{a}}$ & $2 \cdot 4$ & $2 \cdot 5^{\mathrm{b}}$ & 0.4 & $0.2^{c}$ & 0.1 & $2 \cdot 0^{\mathrm{a}, \mathrm{b}}$ & 1.5 & $0.6^{\mathrm{a}}$ & 0.1 & $0 \cdot 1^{b}$ & 0.05 & $16 \cdot 9^{\mathrm{a}}$ & 4.5 & $4.4^{\mathrm{b}}$ & 0.7 & $0.4^{c}$ & 0.2 \\
\hline (1) & & & & & & & & & & & & & & & & & & \\
\hline Food & $62 \cdot 4^{\mathrm{a}}$ & 3.0 & $26 \cdot 5^{\mathrm{b}}$ & $1 \cdot 7$ & $16 \cdot 3^{\mathrm{c}}$ & $2 \cdot 0$ & $56 \cdot 4^{\mathrm{a}}$ & $4 \cdot 3$ & $20 \cdot 4^{\mathrm{b}}$ & $2 \cdot 1$ & $13 \cdot 1^{\mathrm{b}}$ & 2.5 & $68 \cdot 6^{\mathrm{a}}$ & $5 \cdot 6$ & $32 \cdot 2^{\mathrm{b}}$ & $2 \cdot 2$ & $20 \cdot 5^{\mathrm{C}}$ & 2.6 \\
\hline Food and supplements & $61 \cdot 2^{\mathrm{a}}$ & $3 \cdot 2$ & $25 \cdot 4^{\mathrm{b}}$ & 1.6 & $15 \cdot 7^{\mathrm{c}}$ & $2 \cdot 0$ & $55 \cdot 0^{\mathrm{a}}$ & 4.1 & $19 \cdot 7^{\mathrm{b}}$ & $2 \cdot 1$ & $12 \cdot 6^{\mathrm{b}}$ & $2 \cdot 4$ & $67 \cdot 4^{\mathrm{a}}$ & 5.4 & $31 \cdot 3^{\mathrm{b}}$ & $2 \cdot 2$ & $19 \cdot 8^{\mathrm{C}}$ & $2 \cdot 6$ \\
\hline P & & & & & & & & & & & & & & & & & & \\
\hline Food & $31 \cdot 8^{\mathrm{a}}$ & $6 \cdot 0$ & $13 \cdot 6^{b}$ & 1.9 & $8 \cdot 2^{b}$ & 1.4 & $11 \cdot 5$ & 9.3 & $4 \cdot 8$ & $1 \cdot 2$ & $4 \cdot 3$ & $1 \cdot 1$ & $53 \cdot 6^{\mathrm{a}}$ & $7 \cdot 5$ & $21 \cdot 6^{\mathrm{b}}$ & 3.2 & $13 \cdot 5^{\mathrm{b}}$ & $2 \cdot 8$ \\
\hline Food and supplements & $31 \cdot 1^{\mathrm{a}}$ & $5 \cdot 1$ & $13 \cdot 1^{\mathrm{b}}$ & $1 \cdot 8$ & $8.0^{\mathrm{b}}$ & $1 \cdot 2$ & $11 \cdot 2$ & 8.0 & $4 \cdot 7$ & 1.2 & $3 \cdot 8$ & 1.0 & $52 \cdot 1^{\mathrm{a}}$ & $8 \cdot 3$ & $20 \cdot 8^{\mathrm{b}}$ & 3.1 & $13 \cdot 2^{\mathrm{b}}$ & $2 \cdot 7$ \\
\hline $\mathrm{Zn}$ & & & & & & & & & & & & & & & & & & \\
\hline Food & $23 \cdot 0^{\mathrm{a}}$ & $6 \cdot 1$ & $6 \cdot 8^{\mathrm{b}}$ & 1.9 & $5.0^{\mathrm{b}}$ & 1.4 & $11 \cdot 3$ & 5.1 & 2.5 & 0.8 & 2.5 & 1.2 & $36 \cdot 4^{\mathrm{a}}$ & 10.0 & $10 \cdot 8^{\mathrm{b}}$ & 3.4 & $8 \cdot 6^{\mathrm{b}}$ & $2 \cdot 2$ \\
\hline Food and supplements & $21 \cdot 9^{\mathrm{a}}$ & $5 \cdot 4$ & $6 \cdot 4^{b}$ & 1.8 & $4 \cdot 9^{b}$ & 1.2 & 11.4 & 5.6 & $2 \cdot 4$ & 0.8 & $2 \cdot 4$ & $1 \cdot 1$ & $34.3^{\mathrm{a}}$ & $9 \cdot 1$ & $10 \cdot 4^{\mathrm{b}}$ & 3.2 & $8 \cdot 3^{\mathrm{b}}$ & $2 \cdot 1$ \\
\hline
\end{tabular}

RTEC, ready-to-eat cereal included with breakfast.

etters were significantly different $(P<0.05$; Bonferroni-adjusted $P<0.0167)$

ability method was used. Usual intakes were determined using the Cycle 2.2. The prevalence of inadequate 
in breakfast non-consumers than in RTEC-breakfast consumers, neither of which differed from other-breakfast consumers. Also, for food and total intakes of thiamin, riboflavin, niacin and vitamin $B_{12}$, there were no significant group differences in the prevalence of nutrient inadequacy.

The prevalence of nutrient inadequacy cannot be determined for nutrients that have an adequate intake (AI) instead of an EAR, but the proportion with intakes above the AI can be considered adequate ${ }^{(13)}$. Over $99 \%$ of all the three breakfast groups had $\mathrm{Na}$ intakes above the $\mathrm{AI}$, with no significant differences among the groups. In contrast, although significantly higher proportions of both groups of breakfast consumers met the AI for fibre compared with breakfast non-consumers, the proportions were $<2 \%$ for all the three groups. A somewhat similar situation existed for $\mathrm{K}$, with 0.8 (SEM 0.6 ) $\%$ of breakfast non-consumers, 4.9 (SEM 0.7 ) \% of other-breakfast consumers and $7 \cdot 0$ (SEM $2 \cdot 4$ ) \% of RTECbreakfast consumers meeting the AI. These data are based on intakes from food sources alone, as only trivial amounts of $\mathrm{Na}$, fibre and $\mathrm{K}$ were consumed as supplements.

\section{Potential risk of excessive intakes}

With the exception of $\mathrm{Na}$ and $\mathrm{Zn}$, the prevalence of the potential risk of excessive intakes (above the UL) for all three breakfast groups was $<1 \%$ from food sources alone and $<2.5 \%$ from the combination of food sources plus supplements. There were no differences among the breakfast groups. For $\mathrm{Na}$, the prevalence of intakes above the UL from food sources only was approximately $90 \%$, and did not differ by breakfast group. The proportions of $\mathrm{Zn}$ intakes above the UL from food sources alone were 0.7 ( 4.8 (SEM 0.9 ) and 8.0 (SEM 1.8 ) \% for breakfast non-consumers, other-breakfast consumers and RTEC-breakfast consumers, respectively. From food sources plus supplements, the proportions of $\mathrm{Zn}$ intakes above the UL were 1.3 (SEM 0.8 ), 5.7 (Sем 0.9) and 8.8 (SEм 1.8)\%. In both cases, breakfast non-consumers had a significantly lower prevalence than the two groups of breakfast consumers, which did not differ.

\section{Discussion}

Underconsumed nutrients of potential public health concern in Canadian children and/or adolescents include $\mathrm{Ca}, \mathrm{P}, \mathrm{Mg}$, $\mathrm{K}$, vitamin A, vitamin D and fibre ${ }^{(31,32)}$. For these shortfall nutrients, intakes in the present study increased in a stepwise manner among breakfast non-consumers, other-breakfast consumers and RTEC-breakfast consumers (Ca, P, Mg, K, vitamin $\mathrm{D}$ and fibre) or were higher in both groups of breakfast consumers than in breakfast non-consumers (vitamin A). These findings are consistent with those of previous studies in other countries ${ }^{(5-11,33)}$. Of greater relevance than higher intakes, however, is our finding of stepwise decreases in the prevalence of dietary inadequacy for $\mathrm{Ca}, \mathrm{Mg}, \mathrm{Fe}$ and vitamin D among breakfast non-consumers, other-breakfast consumers and RTEC-breakfast consumers. This was the case for intakes from food sources alone and from the combination of food sources plus supplements. Furthermore, compared with breakfast non-consumers, the two groups of breakfast consumers had a lower prevalence of inadequacy for vitamin $\mathrm{A}$ and $\mathrm{P}$ (food sources alone and food sources plus supplements) and vitamin C (food sources plus supplements). Thus, breakfast consumption, and to a greater extent, RTEC breakfast consumption, was associated with meaningful decreases in inadequacy for key nutrients. Moreover, although the prevalence of inadequacy cannot be estimated for fibre and $\mathrm{K}$, higher proportions of breakfast consumers met the AI for these nutrients.

In addition to the aforementioned shortfall nutrients, breakfast consumers had significantly higher intakes of several other nutrients. However, unlike the improvements in nutrient adequacy for the shortfall nutrients, higher intakes of the other nutrients did not always translate into significantly improved nutrient adequacy. In some cases, this was due to large standard errors (e.g. folate inadequacy was more prevalent in breakfast non-consumers than in breakfast consumers, but the difference was not significant). In other cases, it was related to the fact that the requirement is a cut-off point: when intakes of almost all the participants in a group exceed the EAR (e.g. riboflavin and niacin in breakfast nonconsumers), higher intakes do not reduce the prevalence of inadequacy. Conversely, if a relatively large proportion has intakes near the requirement, small increments can substantially change the proportion that do (or do not) meet their requirements. This was the case for $\mathrm{Mg}$ in the present study, where relatively modest increases in intake were associated with substantial reductions in inadequacy between breakfast non-consumers, other-breakfast consumers and RTEC-breakfast consumers. These outcomes illustrate the importance of assessing nutrient adequacy, rather than focusing primarily on differences in nutrient intakes among groups.

In contrast to the impact of breakfast consumption on nutrient adequacy, supplement use did not appear to substantially reduce the prevalence of nutrient inadequacy, as can be seen by the generally similar prevalences of inadequacy from food sources alone $v$. from food sources plus supplements (Table 4). The exception was vitamin $\mathrm{D}$, where the prevalence of nutrient inadequacy was 10 to 20 percentage points lower when supplement use was considered. However, it should be noted that the EAR for vitamin $\mathrm{D}$ reflects the estimated requirement for dietary intake in the absence of sunlight exposure $^{(34)}$. Although Canadian children have an apparently high prevalence of low vitamin D intakes (irrespective of supplement use), assessment of the vitamin D status of Canadian children and adolescents using serum 25-hydroxyvitamin D does not suggest widespread vitamin D deficiency ${ }^{(35)}$. This indicates that the contribution of sunlight exposure is probably substantial.

$\mathrm{Zn}$ was the only nutrient for which the prevalence of the potential risk of excessive intakes was higher in breakfast consumers than in non-consumers. Intakes above the UL occurred almost exclusively in children aged 4 to 8 years (data not shown). In both Canada and the USA, the mean $\mathrm{Zn}$ intakes of children aged 1 to 3 years exceed the UL, and considerable proportions of children aged 4 to 8 years exceed the $\mathrm{UL}^{(29,36-38)}$. Based on these high prevalences, the absence of 
any evidence of adverse effects and the limited data used to set the UL, it has been suggested that the UL for $\mathrm{Zn}$ for young children was set at too low a level ${ }^{(39)}$. Further research is needed to better define a UL for children, but in the meantime efforts to reduce $\mathrm{Zn}$ intakes do not appear to be warranted.

Intakes of fat, saturated fat and cholesterol in RTECbreakfast consumers appeared to be more closely aligned with current recommendations than were intakes of breakfast non-consumers or other-breakfast consumers. However, RTEC-breakfast consumers also consumed approximately $10 \mathrm{~g} / \mathrm{d}$ more total sugars (the CCHS 2.2 data file does not differentiate between naturally occurring and added sugars). The difference in sugar intake occurred at the breakfast meal, since intakes during the rest of the day were virtually identical between the RTEC-breakfast consumers and otherbreakfast consumers. Since approximately $95 \%$ of those who consume RTEC at breakfast consume it with milk ${ }^{(40)}$, and $\mathrm{Ca}$ intake at breakfast averaged $120 \mathrm{mg}$ higher among the RTEC-breakfast consumers than among the other-breakfast consumers, about half the higher sugar intake would have come from milk (120 mg Ca is provided by approximately $100 \mathrm{ml}$ of milk, which contains approximately $5 \mathrm{~g}$ of lactose). Accordingly, the remaining difference (approximately $5 \mathrm{~g}$ ) was probably added sugars provided by RTEC and/or the addition of sugar to cereal at the table. Some debate exists regarding the adverse effects of added sugars on health ${ }^{(41-44)}$; nevertheless, dietary guidance in both Canada $^{(45)}$ and the $\mathrm{USA}^{(46)}$ recommends that intakes be reduced. While the entire food supply, including RTEC, warrants examination, the highest priority for reduction would appear to be foods/beverages with large amounts of added sugars and without meaningful amounts of other nutrients.

The strengths of the present study include a large sample size, use of rigorous methodology to examine nutrient adequacy, and consideration of supplement use. Furthermore, our data indicate that food choices at breakfast per se make important contributions to overall nutrient intake and adequacy, as most differences in daily intakes between those consuming RTEC $v$. other types of breakfasts were due to the intake at the breakfast meal (rather than during the rest of the day). These differences were probably due to nutrients present (or added as fortificants) in RTEC and the milk that normally accompanies it.

We also acknowledge a number of limitations. First, breakfast groups were defined based on intake on the day of the first $24 \mathrm{~h}$ dietary recall. Whether or not those classified as breakfast non-consumers, RTEC-breakfast consumers and other-breakfast consumers followed these specific breakfast patterns routinely could not be ascertained. However, the fact that a number of demographic characteristics differed among the three breakfast groups suggests some stability in the classification. In this regard, it should also be noted that variability in breakfast patterns would attenuate the observed group differences, rather than exaggerate them. A second limitation is that the potential for residual confounding cannot be excluded. In this regard, physical activity, 'screen time' and smoking could not be included as covariates in the primary analysis of nutrient intake. However, the pattern of differences among the breakfast groups was almost identical when these covariates were added to separate analyses of those aged 6 to 11 and 12 to 17 years, suggesting that their omission from the primary analysis did not have a meaningful impact on the results. Third, we also note that we assessed only two types of breakfasts: those that included or did not include RTEC. Other breakfast patterns may also be associated with variability in nutrient intakes and adequacy, and this warrants additional research. Finally, food intake data were selfreported.

\section{Concluding remarks}

The results of the present study support our hypothesis that breakfast consumption in Canadian children and adolescents would be associated with a lower prevalence of nutrient inadequacy, and would have little or no impact on the prevalence of the potential risk of adverse effects from excessive nutrient intakes. A key finding was that improved adequacy was seen for nutrients of potential public health concern, including $\mathrm{Ca}, \mathrm{Mg}, \mathrm{P}$, vitamin $\mathrm{A}$ and vitamin $\mathrm{D}$, as well as higher intakes of $\mathrm{K}$ and fibre. Breakfasts that included RTEC were associated with further improvements for several of these nutrients, while also adding approximately $5 \mathrm{~g}$ to added sugar intake. The prevalence of skipping breakfast increased substantially with age. Given the impact on nutrient intake and adequacy, efforts to encourage and maintain breakfast consumption are warranted.

\section{Acknowledgements}

The authors thank Carmina Ng and Dave Haans at the Statistics Canada Toronto Region Research Data Centre for their support in accessing the CCHS 2.2 data file. While the research and analysis are based on data from Statistics Canada, the opinions expressed do not represent the views of Statistics Canada.

The present study was supported by Kellogg Canada, Inc. The funder had no role in the design and analysis of the study or in the writing of this article.

The authors' responsibilities were as follows: S. I. B., L. D. and V. L. F. designed the study; V. L. F. was primarily responsible for the data analysis; S. I. B. wrote the first draft of the manuscript; L. D. and V. L. F. provided critical input and contributed to the writing of the manuscript. All authors read and approved the final version of the manuscript.

At the time the research was conducted, S. I. B. and L. D. had consulting agreements with Kellogg Canada, Inc. V. L. F., as President of Nutrition Impact, LLC performs consulting and database analyses for various food and beverage companies and related entities.

\section{References}

1. Rampersaud GC (2009) Benefits of breakfast for children and adolescents: update and recommendations for practitioners. Am J Lifestyle Med 3, 86-103. 
2. Rampersaud GC, Pereira MA, Girard BL, et al. (2005) Breakfast habits, nutritional status, body weight and academic performance in children and adolescents. J Am Diet Assoc 105, 743-760.

3. Chao E \& Vanderkooy P (1989) An overview of breakfast nutrition. J Can Diet Assoc 50, 225-228.

4. Miller G, Forgac T, Cline T, et al. (1998) Breakfast benefits children in the US and abroad. J Am Coll Nutr 17, 4-6.

5. Deshmukh-Taskar PR, Nicklas TA, O'Neil CE, et al. (2010) The relationship of breakfast skipping and type of breakfast consumption with nutrient intake and weight status in children and adolescents: The National Health and Nutrition Examination Survey 1999-2006. J Am Diet Assoc 110, 869-878

6. Grieger JA \& Cobiac L (2012) Comparison of dietary intakes according to breakfast choice in Australian boys. Eur J Clin Nutr 66, 667-672.

7. Williams BM, O'Neil CE, Keast DR, et al. (2009) Ready-to-eat cereal breakfasts are associated with improved nutrient intake and dietary adequacy but not body mass index in black adolescents. Am J Lifestyle Med 3, 500-508.

8. Van den Boom A, Serra-Majem L, Ribas L, et al. (2006) The contribution of ready-to-eat cereals to daily nutrient intake and breakfast quality in a Mediterranean setting. $J$ Am Coll Nutr 25, 135-143.

9. Gibson SA \& O'Sullivan KR (1995) Breakfast cereal consumption patterns and nutrient intakes of British schoolchildren. J R SOc Promot Health 115, 366-370.

10. Preziosi P, Galan P, Deheeger M, et al. (1999) Breakfast type, daily nutrient intakes and vitamin and mineral status of French children, adolescents and adults. J Am Coll Nutr 18, 171-178.

11. Williams P (2007) Breakfast and the diets of Australian children and adolescents: an analysis of data from the 1995 National Nutrition Survey. Int J Food Sci Nutr 58, 201-216.

12. Siega-Riz AM, Popkin BM \& Carson T (1998) Trends in breakfast consumption for children in the United States from 1965 to 1991. Am J Clin Nutr 67, 748S-756S.

13. Food and Nutrition Board \& Institute of Medicine (2000) Dietary Reference Intakes: Applications in Dietary Assessment. Washington, DC: National Academy Press.

14. Bailey RL, Fulgoni VL 3rd, Keast DR, et al. (2011) Dietary supplement use is associated with higher intakes of minerals from food sources. Am J Clin Nutr 94, 1376-1381.

15. Fulgoni VL 3rd, Keast DR, Bailey RL, et al. (2011) Foods, fortificants, and supplements: where do Americans get their nutrients? J Nutr 141, 1847-1854.

16. Shakur YA, Tarasuk V, Corey P, et al. (2012) A comparison of micronutrient inadequacy and risk of high micronutrient intakes among vitamin and mineral supplement users and nonusers in Canada. J Nutr 142, 534-540.

17. Statistics Canada (2009) Canadian Community Health Survey - Nutrition (CCHS). http://www23.statcan.gc.ca:81/imdb/ p2SV.pl?Function $=$ getSurvey\&SDDS $=5049 \&$ lang $=e n \& d b=$ imdb\&adm $=8 \& d i s=2 \#$ b8 (accessed October 2013).

18. Health Canada (2006) Canadian Community Health Survey Cycle 2.2 Nutrition, 2004. A guide to accessing and interpreting the data. http://www.hc-sc.gc.ca/fn-an/surveill/ nutrition/commun/cchs_guide_escc-eng.php (accessed October 2013).

19. Statistics Canada (2005) Statistics Act 1985, c. S-19 amended by 2005 , c.38. http://www.statcan.gc.ca/about-apercu/actloi-eng.htm (accessed October 2013).

20. Statistics Canada (2013) The Research Data Centres (RDC) program. http://www.statcan.gc.ca/rdc-cdr/index-eng.htm (accessed October 2013).
21. Raper N, Perloff B, Ingwersen L, et al. (2004) An overview of USDA's dietary intake data system. J Food Comp Anal 17, 545-555.

22. Health Canada (2007) The Canadian Nutrient File. http://www.hc-sc.gc.ca/fn-an/nutrition/fiche-nutri-data/cnf_ aboutus-aproposdenous_fcen-eng.php (accessed October 2013).

23. US Department of Agriculture \& Agricultural Research Service (2013) USDA National Nutrient Database for Standard Reference, Release 25. http://www.ars.usda.gov/ba/bhnrc/ ndl (accessed October 2013)

24. Department of Justice (2013) Food and Drug Regulations, Section B.13.060. http://laws-lois.justice.gc.ca/eng/regulations/ C.R.C.\%2C_C._870/page-143.html\#docCont (accessed October 2013).

25. Office of the Federal Register (1993) Nutrition quality guidelines for foods: fortification policy. 58 Federal Register 2228.

26. Tooze JA, Kipnis V, Buckman DW, et al. (2010) A mixedeffects model approach for estimating the distribution of usual intake of nutrients: the NCI method. Stat Med 29, 2857-2868.

27. Center for Disease Control and Prevention \& National Center for Health Statistics (1988) Analytic and Reporting Guidelines: The Third National Health and Nutrition Examination Survey, NHANES III (1998-94) [internet]. Hyattsville, MD: National Center for Health Statistics. http://www.cdc.gov/ nchs/data/nhanes/nhanes3/nh3gui.pdf (cited 14 July 2012).

28. IOM (Institute of Medicine) (1998) Dietary Reference Intakes for Thiamin, Riboflavin, Niacin, Vitamin $B_{6}$, Folate, Vitamin $B_{12}$, Pantothenic Acid, Biotin, and Choline. Washington, DC: National Academy Press

29. IOM (Institute of Medicine) (2001) Dietary Reference Intakes for Vitamin A, Vitamin K, Arsenic, Boron, Chromium, Copper, Iodine, Iron, Manganese, Molybdenum, Nickel, Silicon, Vanadium, and Zinc. Washington, DC: National Academy Press.

30. Garriguet D (2013) Combining nutrient intake from food/ beverages and vitamin/mineral supplements. Health Rep $\mathbf{2 1}$, 71-84. http://www.statcan.gc.ca/pub/82-003-x/2010004/ article/11350-eng.pdf (accessed October 2013).

31. Health Canada (2012) Do Canadian children meet their nutrient requirements through food intake alone? http://www. hc-sc.gc.ca/fn-an/surveill/nutrition/commun/art-nutr-child-enfeng.php (accessed October 2013).

32. Health Canada (2012) Do Canadian adolescents meet their nutrient requirements through food intake alone? http:// www.hc-sc.gc.ca/fn-an/surveill/nutrition/commun/art-nutr-adoleng.php (accessed October 2013).

33. Albertson AM, Anderson GH, Crockett SJ, et al. (2003) Ready-to-eat cereal consumption: its relationship with BMI and nutrient intake of children aged 4 to 12 years. $\mathrm{J} \mathrm{Am}$ Diet Assoc 103, 1613-1619.

34. IOM (Institute of Medicine) (2011) Dietary Reference Intakes for Calcium and Vitamin D. Washington, DC: The National Academies Press.

35. Whiting SJ, Langlois KA, Vatanparast $\mathrm{H}$, et al. (2011) The vitamin D status of Canadians relative to the 2011 Dietary Reference Intakes: an examination in children and adults with and without supplement use. Am J Clin Nutr 91, $128-135$.

36. Butte NF, Fox MK, Briefel RR, et al. (2010) Nutrient intakes of US infants, toddlers, and preschoolers meet or exceed dietary reference intakes. J Am Diet Assoc 110, Suppl. 3, S27-S37.

37. Moshfegh A, Goldman J \& Cleveland L (2005) What We Eat in America, NHANES 2001-2002: Usual Nutrient 
Intakes from Food Compared to Dietary Reference Intakes. Washington, DC: US Department of Agriculture, Agricultural Research Service.

38. Health Canada \& Statistics Canada (2008) Canadian Community Health Survey Cycle 2.2, Nutrition (2004). Nutrient Intakes from Food. Provincial, Regional and National Summary Data Tables, vol. 2, Ottawa, ON: Minster of Health Canada.

39. Zlotkin S (2006) A critical assessment of the upper intake levels for infants and children. J Nutr 136, 502S-506S.

40. Song WO, Chun OK, Kerver J, et al. (2006) Ready-to-eat breakfast cereal consumption enhances milk and calcium intake in the US population. $J$ Am Diet Assoc 106, $1783-1789$

41. Johnson RK, Appel LA, Brands M, et al. (2009) AHA Scientific Statement. Dietary sugars intake and cardiovascular health. Circulation 120, 1011-1020.
42. Ruxton CH, Gardner EJ \& McNulty HM (2010) Is sugar consumption detrimental to health? A review of the evidence 1995-2006. Crit Rev Food Sci Nutr 50, 1-19.

43. Rippe JM (2013) The metabolic and endocrine response and health implications of consuming sugar-sweetened beverages: findings from recent randomized controlled trials. Adv Nutr 4, 677-686.

44. Yang Q, Zhang Z, Greeg EW, et al. (2014) Added sugar intake and cardiovascular diseases mortality among US adults. JAMA Intern Med 174, 516-524.

45. Health Canada (2007) Eating Well with Canada's Food Guide. http://www.hc-sc.gc.ca/fn-an/food-guide-aliment/ index-eng.php (accessed October 2013).

46. US Department of Agriculture \& U.S. Department of Health and Human Services (2010) Dietary Guidelines for Americans, 2010, 7th ed. Washington, DC: U.S. Government Printing Office. 